$$
(i+j-1) ! W_{i+j, 0}=-\left|\begin{array}{cccr}
Q_{10} & -1 & & 0 \\
2 Q_{20} & Q_{10} & -2 & 0 \\
3 Q_{30} & 2 Q_{20} & Q_{10} & -3 \\
\vdots & & & 0 \\
& & & -(i+j) \\
(i+j) Q_{i+j, 0} \ldots & Q_{10}
\end{array}\right|
$$

with a similar expression for $W_{0, i+j}$.

11. Conclusion. It hardly seems necessary to give numerical examples of these expansions. As in the case of recurrents, from expressions of such generality any desired example may be derived by a mere substitution of numbers for letters in the general formulas. The quotient of two polynomials, the reciprocal of a series or a polynomial, for example, are included as special cases.

It appears from the expressions for $Z_{21}$ and $Z_{12}$ in $\$ 7$, that a further immediate reduction of the order of the determinants (17) is sometimes possible; but to explicate this reduction in the general case would be to mar the simplicity and symmetry of our developments.

In conclusion I should like to thank Professor E. T. Bell for criticism and suggestions in the writing of this paper.

California Institute of Technology

\title{
A CORRECTION
}

In the paper by $\mathrm{H}$. W. March, The Heaviside operational calculus, this Bulletin, vol. 33(1927), on page 312, in the line following equation (2), change "negative" to "positive." 